\title{
Moyamoya disease - a review of clinical experience and anaesthetic management
}

\begin{abstract}
Moyamoya disease is a rare neurovascular condition that affects both children and adults. Increasingly these patients present for surgical management to improve the cerebral circulation since medical therapy is essentially ineffective. Because of the precarious cerebral circulation, these patients represent an anaesthetic challenge. In this report we review the pathophysiology of moyamoya disease, summarize our experience with seven patients and discuss the anaesthetic management.
\end{abstract}

Nomura and Takeuchi first described moyamoya disease in 1961., ${ }^{1,2}$ Moyamoya is a Japanese word meaning something hazy like a puff of cigarette smoke drifting in the air! It refers to a characteristic angiographic appearance of this condition and consists of a narrowing or occlusion of both internal carotid arteries at the level of the carotid siphon together with an abnormal haemangiomatosis vascular network at the base of the brain. ${ }^{3}$ These patients often present for neurosurgical treatment to improve cerebral blood flow and constitute an anaesthetic challenge. We have reviewed our clinical experience and summarized the anaesthetic management.

\section{Aetiology and pathophysiology}

The aetiology of moyamoya disease has not been clearly defined. Both congenital and acquired categories have

\section{Key words}

ARTERIES: moyamoya disease; cerebroarteriosynangiosis; COMPLICATIONS: Stroke; ANAESTHETICS, INHALATION: isollurane.

From the Department of Anaesthesia, University Hospital and University of Westem Ontario, London, Ontario.

Address correspondence to: Dr. A. M. Lam, Department of Anaesthesia, Unjversity Hospital, P.O. Box 5339. Postal Stn. A., London, Ontario, N6A 5A5. been proposed. In the congenital form where an arterial dysplastic process involving both cerebral and systemic vasculature has been identified, hereditary factors may be important since seven per cent of the reported cases were familial. The acquired forms could result from a variety of diseases including meningitis, neurofibromatosis, connective tissue disease and chronic inflammation in the neck region. ${ }^{4}$ The pathology of the abnormal vessel is also by no means uniform, although the most prominent features reported have been intimal thickening or deficiency in the internal elastic lamina. ${ }^{4}$

Most of the cases in the literature have come from Japan. This disorder occurs predominantly in children and is much more frequent in girls. The common presentation in children is that of transient ischaemic attacks (TIA's) with the gradual development of fixed neurological deficits. ${ }^{5}$ In adults, the most common mode of presentation is that of intracerebral haemorrhage. ${ }^{6}$

Precipitating factors for TIA's which have been identificd include change in body temperature such as occurring after a hot bath, ${ }^{7}$ hyperpnoca or deep breathing activity associated with vigorous crying spells or singing. ${ }^{8}$

Since 1967, 46 patients with moyamoya disease accompanied by an intracranial aneurysm have been reported. ${ }^{9}$ The incidence of intractanial aneurysms associated with moyamoya disease has been estimated to be 14 per cent in adults, but is rare in childhood. ${ }^{10}$ There are two types of aneurysms associated with moyamoya disease. Saccular aneurysms occur on the Circle of Willis, at the same sites as the general population, but with a different frequency distribution. Instead of occurring in the anterior circulation, aneurysms in this entity are most frequently on the vertebrobasilar system, especially at the basilar bifurcation. The second type is small aneurysms on the peripheral small arteries contributing to the moyamoya vessels or other collaterals. The likely explanation for the increased incidence of posterior circulation aneurysms is obstruction of the internal carotid artery changing the haemodynamics of the cerebral circulation. ${ }^{11}$ 
TABLE Clinical features of moyamoya disease in seven patients

\begin{tabular}{lllll}
\hline Case & Ser & $\begin{array}{l}\text { Age } \\
\text { (years) }\end{array}$ & Presentasion & Past history \\
\hline 1 & M & 30 & Right hemiparesis, aphasia & Left ECIC \\
2 & F & 38 & Right hemiparesis, memory deficit & Basilar aneurysm clipping \\
3 & F & 26 & Leftight hemispheric TIA's, aphasia & Seizures \\
4 & M & 42 & Right hemispheric TIA's, dysarthria, decreased cognition & Right occipital-MCA bypass \\
5 & F & 36 & Left hemiparesis, aphasia, headaches & Left ECIC \\
6 & M & 35 & Left hemispheric TIA's dysarthria, headaches & \\
7 & F & 27 & Right hemiparesis, aphasia &
\end{tabular}

ECIC = extracranial-intracranial anastomosis.

T/A $=$ transient ischaemic attack.

$M C A=$ middle cerebral antery.

\section{Medical management}

Medical management for these patients involves the use of antiplatelet agents such as aspirin that has gained such widespread acceptance for TIA's of atherosclerotic origin. Little else is presently available for non-surgical treatment. The calcium channel antagonist verapamil has been used in several patients ${ }^{12,13}$ with success in reversing acute neurological deficits due to moyamoya disease, presumably on the basis of cerebral vasodilation.

\section{Surgical management}

Surgical correction of this entity had been based on superficial temporal to micdle cerebral artery anastomosis (extracranial-intracranial anastomosis or ECIC) ${ }^{14}$ With the technical difficulty of this procedure in some patients, and the worsening of condition in others after the procedure, a new tecbnique of encephalo-duro-arteriosynangiosis (EDAS) which involves the laying of an intact scalp artery onto the arachnoid surface of the brain to allow development of revascularization was devised. ${ }^{14.15}$ This has changed our preferred operation for moyamoya disease from an ECIC to the EDAS procedure.

\section{Anaesthetic management}

Little has been written on the anaesthetic management of this disease entity. We have retrospectively reviewed uur clinical experience from 1982-1985, in a total of eight procedures (three ECIC and five EDAS) performed on seven patients. The clinical presentation of these patients are summarized in the Table. Partly because of the selection of patients who may benefit from surgery, and partly because of the referral pattern, hemiparesis with TIA's rather than intraccrebral hacmorrhage was the most common mode of presentation in our series.

Of interest in the presenting histories was that six of the seven patients were cigarette smokers. Two patients (\#5 and \#6) were North American Indians, brother and sister, and had a positive family history of cerebral vascular disease. The other five patients were Caucasians.

All patients had angiographic evidence of moyamoya disease. Preoperative laboratory investigation included a complete blood count, serum electrolytes, blood urea nitrogen, creatinine, glucose and urinalysis. An electrocardiogram (ECG), chest $x$-ray, and capillary blood gases were also obtained. Four of the seven patients had abnormal ECG's. Abnormalities included ventricular enlargement and hypertrophy, ST-T wave changes, prenature ventricular contraction, and right and lcft axis deviation. The rest of the laboratory investigations were negative. Three patients were maintained on entericcoated aspirin therapy and one patient with a history of seizures was maintained on diphenylhydantoin. Two patients were treated with propranolol for hypertension.

Inasmuch as this was a retrospective review, there were some variations in the anaesthetic technique employed for these eight procedures. However, because of sub-specialization within the institution, only three anaesthetists were involved in the management of these cases and there was consequently a remarkable uniformity in the approach.

\section{Anaesthetic induction}

Fentanyl was given to all patients at the time of induction $\left(1-3 \mu \mathrm{g} \cdot \mathrm{kg}^{-1} \mathrm{JV}\right.$ ), followed by lidocaine $1-1.5 \mathrm{mg} \cdot \mathrm{kg}^{-1}$ $\mathrm{IV}$, and thiopentone $3-5 \mathrm{mg} \cdot \mathrm{kg}^{-1}$ IV. In seven of the eight procedures, "precurarization" with d-tubocurare $3 \mathrm{mg}$ was followed by succinylcholine $1.5 \mathrm{mg} \mathrm{kg}^{-1} \mathrm{IV}$. In the most recent anaesthetic atracurium $0.5 \mathrm{mg} \cdot \mathrm{kg}^{-1}$ was used to facilitate tracheal intubation.

\section{Maintenance of anaesthesia}

All patients were placed in the supine position with the head turned to one side to allow surgical access. The ECG was monitored in all patients. Intra-arterial catheters were placed in either a radial or dorsalis pedis artery for continuous blood pressure recording. End-tidal $\mathrm{CO}_{2}\left(\mathrm{PeTCO}_{2}\right)$ 
was monitored with a Hewlett Packard capnograph. Nasopharyngeal temperature was monitored and heating blankets were used on all patients to maintain normothermia. No patient's temperature fell below $35^{\circ} \mathrm{C}$. All patients were mechanically ventilated via a Bain circuit, and the $\mathrm{PETCO}_{2}$ was maintained between $37-40 \mathrm{mmHg}$. The intraoperative arterial $\mathrm{PCO}_{2}$ ranged between 35-44 $\mathrm{mmHg}$.

Maintenance of anaesthesia was with low-dose isoflurane (0.5-1.0 per cent inspired) and a nitrous oxide/ oxygen mixture or an air/oxygen mixture $\left(\mathrm{FIO}_{2} 0.35-0.5\right)$, with intermittent doses of pancuronium and fentanyl. The total dose of fentanyl was $150 \mu \mathrm{g}-500 \mu \mathrm{g}$. In the one patient who received atracurium for intubation, muscle relaxation was maintained with atracurium.

With the exception of one patient who had a major complication (see below), anaesthesia was satisfactory and uneventful. In contrast to the labile blood pressure frequently observed during the most common revascularization procedure for cerebral ischaemia, namely, carotid endarterectomy, there was remarkable haemodynamic stability although minor adjustments of isoflurane concentration were often necessary.

The duration of the operation was dependent on the nature of the surgical procedure; it ranged from a minimum of 2 hrs and 25 min for an EDAS to a max imum of 7 hrs for an ECIC.

\section{Intraoperative complications}

Patient \#7 had the only life-threatening intraoperative complication. Thirty minutes after commencement of surgery, she developed premature ventricular contractions (bigeminy) which progressed to transient ventricular tachycardia. This was accompanied by episodic hypertension and hypotension. No apparent aetiological factor could be identified. Her preoperative ECG only showed right axis deviation and non-diagnostic ST changes. Intraoperative blood gases and eletrolytes were normal. Hypotension was treated with phenylephrine, and propranolol was administered to treat the transient ventricular tachycardia. The patient recovered uneventfully.

\section{Emergence}

In all patients neuromuscular blockade was antagonized with atropine $1.2 \mathrm{mg}$ and neostigmine $2.5 \mathrm{mg}$ at the end of the procedure. Lidocaine $1 \mathrm{mg} \cdot \mathrm{kg}^{-1}$ IV was administered to five patients to help prevent reaction to the tracheal tube, as well as to decrease the potential rise in blood pressure during extubation. The success of this manoeuvre could not be ascertained from the chart review. Propranolol (1-3 mg) and hydralazine (25-50 mg) were given IV to control tachycardia and hypertension in two patients. With the patients fully awake, all were extubated in the operating theatre, and then transferred to the Recovery Room.

\section{Postoperative course}

Postoperatively four patients had uneventful recovery after five procedures. Of the other three patients, two patients developed seizures in the recovery room which responded to intravenous diphenylhydantoin, and one patient developed transient hemiplegia which resolved in 24 hrs. All patients were discharged between the fifth and the eighth day. No patient developed any new neurological deficit and one patient had significant improvement of the preoperative weakness. Five of the seven patients were discharged on aspirin therapy. No information on longterm follow-up is available.

\section{Diseussion}

The patient with moyamoya disease has an already compromised cerebral circulation. When caring for these patients who are to undergo a neurosurgical procedure, the utmost attention must be given to preserve this circulation. To date, little has been written on the maragement of these patients under general anaesthesia. ${ }^{16,17}$

We have reviewed our experience with seven patients who underwent various neurosurgical procedures for their moyamoya disease. In the preoperative period, blood gases and an ECG should be obtained in addition to the standard haematological and biochemical investigations. It was noted that four of the seven patients had abnormal ECG's and one of them developed a haemodynamically significant rhythm disturbance intraoperatively. Cardiac abnormality therefore should be considered. Although we did not encounter any coagulation problem in our series, patients receiving aspirin should have a bleeding time determined and increased blood loss anticipated. Patients with a history of seizures should be maintained on anticonvulsant therapy perioperatively.

The basic principle in the anaesthetic management should be the preservation of a proper balance between cerebral blood flow and oxygen consumption. The goal of. the anaesthetic induction was therefore to maintain stable systemic as well as cerebral haemodynamics. Although there was minor variation in the anaesthetic technique used, the basic approach was remarkably uniform. Intubation in seven of eight procedures was facilitated with succinylcholine. In patients with established hemiplegia, a non-depolarizing muscle relaxant should be substituted to avoid possible massive increases in serum potassium. In the most recent procedure, atracurium was used to facilitate intubation as well as to maintain muscle relaxation. Atracurium would be a logical alternative, since it is associated with minimal cardiovascular side-effects. ${ }^{18}$ 
We chose isoflurane for anaesthetic maintenance because of its potentially beneficial cerebral effects. It is a mild cerebral vasodilator, but a potent depressant of cerebral metabolic rate ${ }^{19}$ and may have a cerebral protective effect against ischaemia. ${ }^{20} \mathrm{~A}$ more potent cerebral vasodilator such as halothane may increase $C B F$, but may also cause a "steal" from the already ischaemic areas. However, successful use of halothane has been reported in one case. ${ }^{17}$ Nitrous oxide can also cause cerebral vasodilation and no clear concensus exists regarding its use in neuroanaesthesia. ${ }^{21}$ It is our opinion that nitrous oxide can probably be safely employed. Other altenative techniques may also be acceptable, provided that the basic principle of preserving cerebral and systemic haeodynamic stability is followed. With the increasing trend towards surgical management, there is a need for a prospective trial to determine the optimum anaesthetic management for patients with moyamoya disease.

As mentioned above, moyamoya disease is not infrequently associated with cerebral aneurysms. One patient in our series underwent clipping of a basilar aneurysm prior to the revascularization procedure. In this circumstance meticulous control of blood pressure is required, since sudden hypertension may cause aneurysmal rupture while hypotension may precipitate ccrebral ischaemia.

Intraoperatively, we believe that monitoring should include ECG, intra-arterial blood pressure measurement, temperature and $\mathrm{PeTCO}_{2}$. The intra-arterial catheter allows not only continuous monitoring of blood pressure but also frequent blood gas analysis.

Because changes in body temperature have been reported to be one of the factors precipitating transient ischaemia, we felt that it was important to maintain the body temperature as close to normal as possible, with the use of heating blankets.

PetCO ${ }_{2}$ monitoring is essential because of the profound influence of $\mathrm{CO}_{2}$ on the cerebral circulation. As demonstrated by ${ }^{133} \mathrm{Xe}$ inhalation, most patients with moyamoya disease show low values of mean hemispheric blood flow in both hemispheres. ${ }^{22}$ Regional cerebral blood flow was reduced uniformly by hyperventilation. Hypoventilation caused regional cerebral blood flow to increase in the temporo-occipital regions but to decrease or remain unchanged in the frontal region. This effect of $\mathrm{CO}_{2}$ is further substantiated by the classic finding of the EEG pattern - the occurrence of prominent high voltage delta bursts following hyperventilation, with a slow return to the normal pattern. ${ }^{23}$ Since Takeuchi ${ }^{22}$ has shown that both hyper- and hypocapnia detrimentally affect the cerebral circulation, normocapnia should be maintained. Sumikowa also suggested that intraoperative hypocapnia may cause neurological deterioration. ${ }^{16}$ Binghan $^{17} \mathrm{re}$ cently reported a case of a 21 -month-old child with moya- moya disease undergoing three uneventful general anaesthetics with spontaneous ventilation, and speculated that the patient's initial neurological deterioration following intubation for inspiratory stridor was caused by hypocapnia secondary to mechanical ventilation. The lack of neurological deterioration associated with the maintenance of normocapnia in our series supports the earlier conclusions. Although it would be desirable to monitor cerebral function intraoperatively, ejther with a computer-processed EEG or somatosensory evoked potentials, the usefulness of such monitors for these procedures remains to be established.

Postoperatively, as demonstrated in our series, patients with moyamoya disease are prone to the development of seizures. Precautions must therefore be taken inducing appropriate nursing protocols and continuation of anticonvulsant therapy. In addition, new neurological deficits may develop and these should be carefully observed to rule out surgically correctable causes.

In summary, when managing patients with moyamoya disease under general anaesthesia special attention must be paid to minimizing changes in $\mathrm{CBF}$. The importance of maintenance of normothermia and normocapnia is emphasized.

\section{References}

1 Nomura $T$. Atlas of cercbral angiography. Tokyo: Sgakushoin, 1961; 192-5.

2 Takeuchi S, Kabayashi K, Tsuchida T et al. Computed tomography in moyamoya disease. J Comput Assist Tomogr 1982; 6: 24-32

3 Savit JM, Levy LA, Reiner MA, Freed IS. Moyamoya disease. NY State J Med 1983; 83: 237-9.

4 Suzuki I, Kodama N. Moyamoya disease - a review. Stroke $1983 ; 14: 104-9$.

5 Golden GS. Strcke syndromes in childhood. Neurological Clinics 1985; 3: 59-75.

6 Aoki $N$, Mizutani $H$. Does moyamoya discase cause subarachnoid hemorrhage? Revicw of 54 cases with intracranial hemorrhage confirmed by coniputerized tomography. J Neurosurg 1984; 60: 348-53.

7 Kurokawa T, Chen YJ, Tamita S, Kishikawa T, Kitamura $K$. Cerebrovascular occlusive disease with and without the moyamoya vascular network in children. Neuropediatrics $1985 ; 16: 29-32$.

8 Fukuyama $Y, U$ mezu $R$. Clinical and cerebral angiographic evolutions of idiopathic progressive occlusive disease of the circle of Willis ("moyamoya" disease) in children Brain Dev 1985; 7: 21-27.

9 Konishi Y, Kadowaki C, Hara M, Takeuchi K. Aneurysms associated with moyamoya disease. Neurosurgery 1985; 16: 484-91. 
10 Waga S, Tochio $H$. Intracranial aneurysm associated with moyamoya disease in childhood. Surg Neurol 1985; 23: $237-43$.

11 Yabumoto M. Funahashi K, Fujii T, Hayashi S, Komai N Moyamoya disease associaled with intracranial aneurysms. Surg Neurol 1983; 20: 20-4.

12 McLean MJ, Gebarski SS, van der Spek AFL, Goldstein $G W$. Response of moyamoya disease to verapamil. Lancer 1985; 1: 163-4.

13 MeLean MJ, Gebarski SS, van der Spek AFL, Goldstein GW. Treatment of acute deficits of moyamoya discase with verapamil. Ann Acad Med (Singapore) 1985; 14: $65-70$.

14 Balagura S, Farris WA. Treatment of moyamoya disease by cerebroarteriosynangiosis. Surg Neurol 1985; 23 . $270-4$.

15 Malsushima $Y$, Suzzki $R$, Tomita $H$, Inaba $Y$. Encephaloduro-arterio-synangiosis (EDAS): a new surgical treatment for chronic cerebral ischemia of childhood-experiences in acute hemiplegia of childhood. 8th Congress of Neurolngical Surgery (WFNS). July 7-13, 1985. Toronto, Abstracts No. 324

16 Strmikawa K, Nagai H. Moyamoya disease and anesthesia (Letter to the Editor). Ancsthesiology 1983; 58: 204-5.

17 Bingham RM, Wilkinson DJ. Anaesthetic management of moya-moya disease. Anaesthesia 1985; 40: $1198-1202$.

18 Basta SJ, Ali HH. Savarese JJ et al. Clinical pharmacology of atracurium besylate (BW 33A): a new nondeplarizing muscle relaxant. Anesth Analg 1982; 61: 723-29.

19 Cucchiara RF, Theye RA, Michenfelder JD. The effects of isoflurane on canine cerebral metabolism and blood flow. Anesthesiology 1974; 40: 571-4.

20 Newberg LA, Michenfelder JD. Cerebral protection by isoflurane during hypoxcmia or ischemia. Anesthesiology 1983; 59: 29-35.

21 Frost EAM. Central nervous system effects of nitrous oxide. In: Eger El (ed). Nitrous Oxide $\mathrm{N}_{2} \mathrm{O}$. New York; Elsevier Science Publishing Co. Inc., 1984; 164.

22 Takeuchi S, Tanaka R, Ishii $R, T$ stuchida $T$, Kobayashi $K$, Arai $H$. Cerebral hemodynamics in patients with moyamoya disease. Surg Neurol 1985; 23: 468-74.

23 Vamashiro $Y$, Takahashi H, Takahashi K. Cerebrovascular moyamoya disease. Eur J Pediatr 1984; 142: 44-50.
Rĕsumé

La maladie de moyamoya est une atteinte neurovasculaire rare pouvant atteindre les enfants et les aduhes. Ces patients se présentent de plus en plus pour un traitement chirurgical consistant à améliorer la circulation cérébrale car le traitement médical est essentiellement inefficace. A cause de l'état précaire de la circulation cérébrale ces patients représentent un défi anesthésique. Dans cet exposé nous revayons la parhophysiologie de la maladie de moyamoya, résumons notre expérience de sept patients, et discutons de la conduite anesthésique. 